the brachial artery, which formed a solid cord, with some thickening at the bend of the elbow. The nails and ends of the fingers now began to have a faint purple tinge; and there were two discoloured patches on the back of the hand. The return of blood by the veins at the bend of the elbow is very tardy. The pulse at the other wrist is about 90, and very feeble. The action of the heart is quite natural; the tongue red, and rather dry. A slight muco-purulent discharge from the uterus continues, but there is not any uterine tenderness. She sleeps fairly in the intervals of pain, which is not continuous.

Dec. 3lst. - She is considerably relieved from pain ; the hand and fingers are cold, reddish-purple, and shrivelled; the coldness and discolouration extend nearly to the elbow; no pulsation is to be felt in the brachial artery below the margin of the axillary artery, and there very imperfectly. Pulse at the right wrist 120 . Tongue red, glazed, and aphthous.

Jan. 9th, 1868. - In this interval she had much improved in health, taking food and stimulants in fair quantity. The pain has nearly ceased. The hand and forearm are dry, with a few blisters about the bend of the elbow, and a faintlymarked line of demarcation just above it.

11th, - I amputated the arm about midway between the elbow and shoulder, making two flaps. The brachial artery and vein were blocked up with solid coagulum. Not any artery needed a ligature, and there was scarcely any oozing from the cut surfaces. The edges of the wound were brought together with wire sutures, and dressed with a strip of lint moistened in tepid water. Immediate union took place by adhesion, and henceforth recovery was rapid and uninterrupted.

The arteries in the hand and forearm were blocked up at all points, and the muscles shrunken, and of a dull purple colour.

No doubt in this interesting case the starting-point of the mischief lay in the congested state of the uterine vessels, accompanied probably by an unduly plastic state of the blood, leading to the formation of a clot and embolism of the brachial artery, forming in the whole a good specimen of dry gangrene, independent of injury.

The patient was under the immediate care of Dr. Bishop, of Calne, to whose diligent care and attention throughout her well-doing was greatly to be attributed. Bath.

\section{SUBCUTANEOUS INJECTION OF CURARA FOR CHOREA.}

By R. T. WRIGHT, M.D., F.R.C.S., SURGEON, BENGAL ARMY.

Atrention having been drawn to curara by Mr. Alfred Simson ${ }^{1}$ and by Dr. D. Drummond ${ }^{2}$, notes of the following case may be of interest.

Mr. S. C- , aged seventy-eight, widower, farmer, of Hopton, Shropshire, a tenant of Viscount Hill, was an object of much local sympathy, on account of his suffering from true chorea for three years, which had resisted all sorts of treatment; so I was asked to see him in consultation. I visited him with Dr. John Lyon and Mr. W. Gill, son of Lord Hill's agent, and found him almost worn out by jactitations so constant and so violent that he was thrown off his bed, unless protected by railings on every side. $\mathrm{He}$ conld not walk, and his speech was quite unintelligible, excent that a few monosyllables, such as "tea," "no," "here," were shouted out with a painful effort. His spirits were very low, he had great difficulty in swallowing, and he could scarcely ever fall asleep without a dose of chloral hydrate, of which he was very fond, as he was quiet only when asleep. Fortunately his appetite was very good and his bowels regular, though he had no teeth and he was always eating sweets. It seems his family is subject to chorea; several relations had it at various times of life, and his mother, who died at the age of eiohty-five, is said to have suffered from chorea for several years before her death. He was so restless that it was impossible to ascertain his pulse, respiration, or temperature ; his urine on examina-

1 THE LAYCeT, Mag 25th, 1878 , p. 776 .

2 British Medical Journal, June 15 th, 1878, p. 857. tion was found to be clear, neutral, of sp. gr. 1010, free from albumen or casts, but it threw down a faint cloud of phosphates with heat, which readily dissolved with a little nitric acid.

As curara had been recommended in hydrophobia, I suggested it might be tried in chorea, so solutions of the following strength were injected subcutaneously on the dates specified. Jan. 21st, 1878, one-twentieth of a grain; Jan, 22nd, one-twentieth of a grain; Jan. 26th, one-tenth of a grain; Jan. 28th, two-tenths of a grain; Jan. 31st, four-tenths of a grain.

On Feb. 2nd four-tenths of a grain of curara were injected. Pulse (felt for the first time) 68, regular. The jactirations were now far less violent, and both the patient and his relatives remarked the improvement without any bad effect. Four-tenths of a grain were injected on Feb. 4th, 5th, 6th, 7 th, 8th, 9th, and 10th, by which date he was perfectly quiet, able to walk without help, and could sleep at night without chloral. He had no other medicine at all but curara since the subcutaneous injection treatment was begun. Fourtenths of a grain were injected on the 14th and 18th; on the latter date he spoke a sentence, slowly, but plainly, and readily intelligible by listening attentively. On the 19th four-tenths of a grain were injected, the patient being in remarkably good spirits. The same quantity was injected on the 22nd, on which day Mr. C- put on his spectacles and read his Bible-the first time he had been able to do so for three years.

From the last date he has been kept quiet by injecting four-tenths of a grain of curara twice a week. His speech is still of abnormal character; but he will not submit to the operation more than twice a week, as he has a morbid dread of the pain, and often breaks the nozzle of the syringe by his frantic struggles when the doctor approaches him. His relatives say he always, even when in health, had a morbid dread of pain, never would wash in cold water, and howled piteously at the slightest scratch. He was sent to Matlock at the outset of his treatment to have proper shower-baths, but he would not stay a week, as he dreaded the water.

The preparation used in Mr. C___'s case was by Messrs. Savory and Moore, in the form of discs of gelatine, a quarter of an inch square. It is made of three strengths, in which each disc contains respectively one-hundredth of a grain, one-twentieth of a grain, and one-tenth of a grain, so that it is easy to obtain a solution of any desired strength. A few drops of water are placed in a spoon; then the discs required for use are added, and the fluid is warmed over a lighted taper; stirring, if necessary, to assist solution. It is necessary to clean the syringe with warm water directly after use, as curara is very sticky, and solidifies when cold so rapidly that it is apt to block up the nozzle of the instrument.

Parliament-street, S.W.

\section{EXTRACTION OF STEEL AND IRON FROM THE EYE BY THE MAGNET.}

BY W. A. M'KEOWN, M.D.,

SURGEON TO THE ULSTER EYE, EAR, AND THROAT HOSPITAL.

THE following cases will doubtless be of interest, taken in conjunction with that not long ago brought before the Clinical Society of London by Mr. M'Hardy.

Dawson B-, aged twenty-four, smith's helper, applied to me at the hospital on Jan. 16th, 1877. He stated that three days previously his right eye had been wounded by a small piece of metal. I observed that the iris was attached to the lens at the outer part of the pupil by recent lymph, and that there was a limited opacity of the lens. There was a small clear metallic body sticking at the margin of the adherent pupil. I made a small section of the cornea, more peripheral than the pupil, introduced a pair of iridectomy forceps, and seized the body and a little piece of iris; but the body slipped from my grasp, and was gliding out of my reach. Fortunately, I had anticipated such an untoward event, and took care to have a pointed permanent magnet at hand. I introduced it into the wound. The metal was instantly attracted and withdrawn. The patient continued under my observation till the 16th February. The opacity of the lens remained limited to the part wounded. I believe the wound in the capsule was closed by lymph and healed. I have not seen nor heard from the patient since. 